\title{
Effectiveness and safety of oxycodone/naloxone in the management of chronic pain in patients with systemic sclerosis with recurrent digital ulcers: two case reports
}

\author{
This article was published in the following Dove Press journal: \\ Clinical Interventions in Aging \\ 14 March 2016 \\ Number of times this article has been viewed
}

\section{Nicola Ughi \\ Chiara Crotti \\ Francesca Ingegnoli}

Division of Rheumatology,

Department of Clinical Sciences and

Community Health, Gaetano Pini

Institute, The University of Milan, Milan, Italy
Correspondence: Francesca Ingegnoli Division of Rheumatology, Department of Clinical Sciences and Community Health, Gaetano Pini Institute, The University of Milan, Piazza Cardinal Ferrari I, Milan 20I22, Italy

Tel +39025829 6456

Fax +390258296604

Email francesca.ingegnoli@unimi.it

\begin{abstract}
Digital ulcers (DUs) are a severe and frequent clinical feature of patients with systemic sclerosis (SSc). The presence of DUs may cause severe pain and often lead to impairment of patient's functional activities and health-related quality of life. Moreover, poor patient cooperation during the wound care procedure due to pain may be associated with a negative outcome of DU healing. Therefore, pain management has a key role in patients with SSc. These two case reports describe the effectiveness and safety of oxycodone/naloxone in patients with SSc complicated by painful chronic DUs. Such a therapy has provided pain relief and consequently an increased compliance during redressing wounds.
\end{abstract}

Keywords: oxycodone, naloxone, systemic sclerosis, pain, digital ulcer, scleroderma, analgaesia, wound healing, opioids, calcinosis, UCLA-SCTC GIT 2.0

\section{Introduction}

Systemic sclerosis (SSc) is a complex multisystem disorder characterized by immune system activation, vascular involvement, and multiorgan fibrosis. ${ }^{1}$ Vasculopathy is postulated to have a key role in the early pathogenesis of SSc and in some of the complications of the disease, such as digital ulcerations.

Digital ulcers (DUs) are a serious and burdensome clinical manifestation of SSc, typically occurring on the fingertips or over the extensor aspects of the hands, and may also be related to calcinosis or become infected. Approximately 50\% of patients report a previous history of DUs, ${ }^{2}$ often occurring early ${ }^{3}$ and predicting a worse disease course. ${ }^{4}$

The presence of DUs accounts for much of the pain in SSc and is significantly associated with disability, ${ }^{5}$ affecting the impairment of daily activities, ${ }^{6}$ reduction in quality of life, ${ }^{7}$ and a negative impact on occupation. ${ }^{8}$ Indeed, patients with SSc report the impact of DUs as worse than other disease manifestations, such as lung and gastrointestinal (GI) problems. ${ }^{9}$

Despite the impact of DUs, there is currently a limited evidence base to guide the management of DUs in patients with SSc. In addition to vasodilator therapy, wound care is pivotal, and sometimes surgical debridement is required (eg, underlying calcinosis), but such procedures may be exquisitely painful. ${ }^{10}$ As pain management has a key role in wound healing and standard therapy (ie, nonsteroidal anti-inflammatory drugs) is often inadequate or dose limited by side effects, opioids are likely to be used. 
However, effective pain management has not been investigated in detail in patients with SSc and the experience in DUs is further limited, particularly regarding opioids such as oxycodone. ${ }^{11,12}$ Due to the lack of best practice consensus, analgesic strategies are not standardized and are mainly guided by a physician's experience.

Against this background, we report the experience of a tertiary referral center concerning the pain management of two patients with SSc affected by DUs with oral prolongedrelease (PR) oxycodone/naloxone (OXN-PR) combination. Such a drug is approved to treat severe chronic pain non responsive to non-opioids. Our institutional review board approval was obtained for publication of this paper.

\section{Case report I}

A 71-year-old woman with a 20-year history of diffuse cutaneous SSc (anti-Scl70 antibodies positive) was admitted for multiple recurrent DUs in August 2015.

Her history was notable for SSc multiorgan involvement (mildly progressive interstitial lung disease previously treated with cyclophosphamide, then ongoing mycophenolate, and stable esophagopathy treated with daily prokinetics, proton-pump inhibitors, and antacids), controlled hypertension, statin-treated hypercholesterolemia, osteoporosis, generalized osteoarthritis, and an extrapyramidal syndrome in follow-up.

New DUs developed in the third fingertip of the left hand and in the second fingertip of the right hand with underlying calcinosis. All DUs had similar aspects: round in shape with a diameter of $4 \mathrm{~mm}$ and a depth of $\sim 2 \mathrm{~mm}$, edges were flat with normal surrounding skin, and bases were pink. In addition, the patient had multiple concurrent scars from the second to the fourth fingertips bilaterally. During admission, swabs collected from all DUs were cultured and the findings were negative.
Full-dose oral calcium antagonist (nifedipine) and bosentan $125 \mathrm{mg}$ twice a day (bid) were regularly administered, and intravenous vasodilator iloprost therapy was intensified to daily administration and carried on as appropriate.

Despite the ongoing analgesic treatment (paracetamol 3,000 mg daily), pain control was poor and wound care was limited by the intense pain. During admission, OXN-PR $5 / 2.5 \mathrm{mg}$ bid was started. At baseline and after 2 months prior to wound care, pain and GI symptoms were assessed. The Brief Pain Inventory was used to assess the severity of pain and the impact of pain on daily functions. Based on a verbally administered 0 - to 10 -point numeric rating scale, ${ }^{13}$ the arithmetic mean of the four severity items can be used as measures of pain severity, while the arithmetic mean of the seven interference items can be used as a measure of pain interference (Table 1). Common adverse events of the GI tract related to the opioid therapy were monitored using the validated Italian version of UCLA Scleroderma Clinical Trial Consortium Gastrointestinal Tract (UCLA-SCTC GIT) 2.0. ${ }^{14}$ UCLA-SCTC GIT 2.0 is a 7-multi-item scale with areas of reflux, distention/bloating, diarrhea, fecal soilage, constipation, emotional well-being, and social functioning. Each item is scored from 0 to 3 except the diarrhea and constipation (range from 0-2 and 0-2.5, respectively), with the lower values indicating a better health-related quality of life. The total UCLA-SCTC GIT 2.0 score averages six of seven scales (excluding constipation) and is scored from 0 (no GI problems) to 3 (most severe). ${ }^{15}$ In this study, we used the total and constipation scores (Table 1).

Throughout the treatments, pain assessment showed a reduced numeric rating scale score in the absence of opioidrelated adverse events, particularly with regard to bowel movements (Table 1). The dose of OXN-PR remained

Table I Assessment of pain and gastrointestinal symptoms using patient-reported outcomes during treatment with oral prolongedrelease oxycodone/naloxone combination

\begin{tabular}{|c|c|c|c|c|}
\hline & \multicolumn{2}{|c|}{ Case report I } & \multicolumn{2}{|c|}{ Case report 2} \\
\hline & Baseline & 2 months later & Baseline & 2 months later \\
\hline \multicolumn{5}{|l|}{ Drug and dosage } \\
\hline OXN-PR daily dose (mg) & $5 / 2.5$ bid & $5 / 2.5$ bid & $5 / 2.5$ bid & $5 / 2.5$ bid \\
\hline \multicolumn{5}{|l|}{ Pain assessment } \\
\hline Pain intensity, mean NRS score & $5 / 10$ & $3 / 10$ & $8 / 10$ & $4 / 10$ \\
\hline $\mathrm{BPI}$, severity & $24 / 50$ & $14 / 50$ & $39 / 50$ & $19 / 50$ \\
\hline $\mathrm{BPI}$, interference & $40 / 50$ & $22 / 50$ & $34 / 50$ & $27 / 50$ \\
\hline \multicolumn{5}{|l|}{ GIT symptom assessment } \\
\hline UCLA-SCTC GIT 2.0 total score & 0.58 & 0.43 & 0.06 & 0.83 \\
\hline UCLA-SCTC GIT 2.0 constipation score & 1.0 & 0.5 & 0.0 & 0.0 \\
\hline
\end{tabular}

Note: Bid means twice a day.

Abbreviations: BPI, Brief Pain Inventory; GIT, gastrointestinal tract; NRS, numeric rating scale; OXN, oxycodone/naloxone; PR, prolonged release; UCLA-SCTC GIT 2.0, UCLA Scleroderma Clinical Trial Consortium Gastrointestinal Tract 2.0. 
stable. Residual pain was reported only during ulcer dressing procedures, but compliance to wound care significantly ameliorated.

\section{Case report 2}

A 61-year-old woman with a 19-year history of limited cutaneous $\mathrm{SSc}$ (anticentromere antibodies positive) was admitted to our Rheumatology Unit for multiple recurrent infected DUs with underlying calcinosis.

Her history was notable for SSc-related esophagopathy (ongoing daily prokinetics, proton-pump inhibitors, and antacids), active smoking with moderate chronic obstructive pulmonary disease (ongoing inhaled bronchodilators), chronic obstructive peripheral arteriopathy (ongoing cilostazol), statin-treated hypercholesterolemia, and osteoporosis (ongoing supplementation of calcium plus vitamin D).

Standard treatment for multiple recurrent DUs accounted for oral calcium antagonist (amlodipine), bosentan $125 \mathrm{mg}$ bid, intravenous iloprost, and human immunoglobulin therapy every other month. For multiple calcinosis and recurrent DU infections, the patient underwent multiple surgical debridements and repeated courses of antibiotics according to antibiograms as appropriate.

In September 2015, DUs developed again in the fourth fingertip of the left hand and in the third and fifth fingertips of the right hand with an underlying calcinosis (Figure 1).

At the fourth fingertip of the left hand, DU was round in shape with a diameter of $4 \mathrm{~mm}$ and a depth of $\sim 3 \mathrm{~mm}$, edges
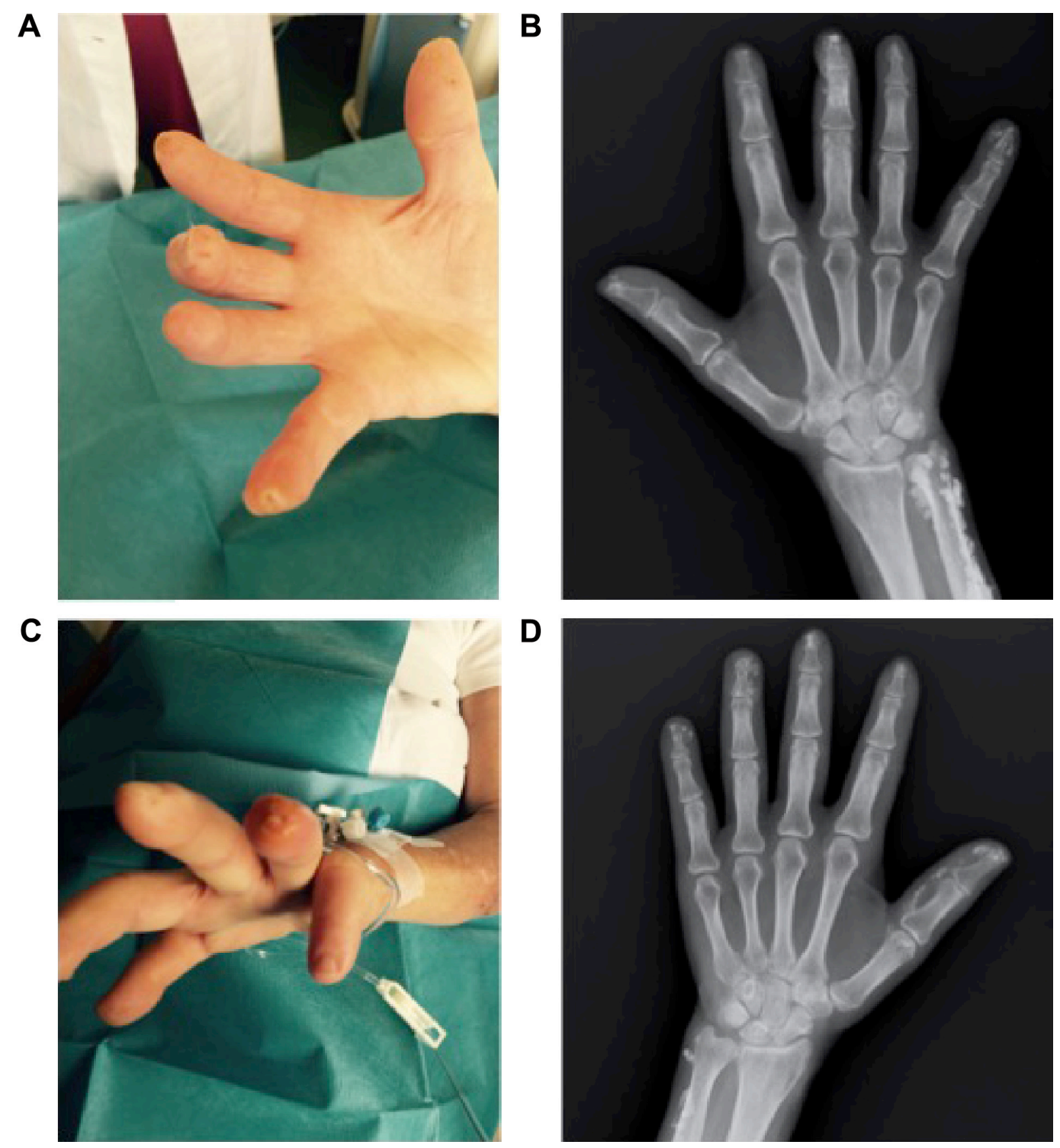

Figure I Digital ulcers of the third and fifth fingertips of the right hand $(\mathbf{A})$ and the fourth fingertip of the left hand $(\mathbf{C})$ at baseline. The underlying calcinosis is shown in the plain X-ray images of the right (B) and left (D) hands. 
were flat with surrounding skin slightly inflamed, and bases were pink and white with an underlying calcinosis. At the third fingertip of the right hand, DU was round in shape with a diameter of $1 \mathrm{~mm}$ and a depth of $\sim 1 \mathrm{~mm}$, edges were flat with normal surrounding skin, and base was pink. DU at the fifth fingertip of the right hand was round in shape with a diameter of $2 \mathrm{~mm}$ and a depth of $\sim 3 \mathrm{~mm}$, edges were flat with normal surrounding skin, and base was pink. In all DUs, there were no discharges suggesting an infection. During admission, swabs collected from all DUs were cultured and the findings were negative.

Iloprost administration and wound dressing procedures were increased (every third day). The ongoing analgesic treatment (paracetamol 3,000 mg daily) was insufficient for pain relief, hence OXN-PR 5/2.5 mg bid was started.

At baseline and after 2 months prior to wound care, the pain was reduced in Brief Pain Inventory domains (Table 1), in the absence of opioid-related adverse events and changes of bowel movements, according to UCLA-SCTC GIT 2.0. ${ }^{14}$ The dose of OXN-PR remained stable. Compliance to wound care ameliorated as well.

\section{Discussion}

In our anecdotal experience, oral administration of a combination of opioid agonist-antagonist OXN-PR was shown to be effective and well tolerated in the management of chronic pain from DUs in SSc.

Opioids have been defined to be effective and safe in the treatment of chronic cancer and noncancer pain, ${ }^{16,17}$ including rheumatic diseases. However, reports on pain treatment in SSc were sporadic and limited to a few opioids, such as oral tramadol and oxycodone; ${ }^{12,18}$ in SSc-related DUs, the use of oxycodone showed efficacy with only mild side effects (itching, nausea, and/or dizziness), including constipation responsive to treatment in $51.7 \%$ of patients. ${ }^{12}$

Therefore, the experience on the use of opioids in patients with SSc is sparse, and concerns about GI adverse events should be taken into account. With particular attention to opioid-induced constipation in chronic noncancer pain, the OXN-PR combination proved to be effective and better tolerated when compared to oxycodone. ${ }^{19}$ Thus, OXN-PR may also be considered in SSc when standard nonopioid therapy is inadequate or limited by side effects and primarily when GI manifestations are a major issue. Our experience in SSc was consistent with previous reports in patients with non-SSc, and the GI tolerability of OXN-PR was excellent. Moreover, in both patients, pain relief was reached with the initial low dose $(5 / 2.5 \mathrm{mg}$ bid) without the need for a dose increase.
In addition to pain relief, pain control is fundamental to optimize wound care. Treatment adherence is, in effect, increased, and the compliance to change of wound dressings ameliorated as well. In our two cases, the treatment with oral OXN-PR allowed a better local management of DUs, especially in the tolerability of deep wound surgical debridement that otherwise was very difficult to carry out without adequate procedural pain management. Moreover, SSc-related calcinosis is often associated with painful DUs as a worsening factor. ${ }^{20}$ In both cases, calcinosis was present; in the past, both patients underwent calcinosis removal, but they did not experience any beneficial long-term effects. In fact, DUs improved quickly but then recurred, including calcinosis.

In addition to oral formulations, topical use of opioids in SSc ulcers was anecdotally reported..$^{21}$ In a recent uncontrolled trial on patients with SSc, in addition to local administration of anesthetics, local (and oral) morphine administration has been shown as a promising tool for the management of procedural pain in wound care of DUs in SSc. ${ }^{22}$ However, topical opioids are not easily accessible and, with regard to our experience, not available for routine use in clinical practice.

\section{Conclusion}

Management of pain related to DUs in SSc is a relevant, yet poorly investigated issue. The choice for optimal analgesia is often challenging, and opioids may be considered when standard therapy is inadequate.

In our anecdotal experience, oral administration of OXN-PR combination is safe and well tolerated in SSc, and it might be considered for DU pain and to optimize the overall treatment of DUs. Due to the lack of evidence base, clinical trials in SSc concerning opioids, especially OXN-PR, are warmly suggested to evaluate their effectiveness and safety in such populations.

\section{Acknowledgment}

The study was completed independently with no funding. Written informed consent was obtained from the patients for the publication of these case reports and accompanying images.

\section{Authors' contributions}

All authors contributed to the acquisition of data and drafting and critically revising the article and agreed to be accountable for all aspects of the work. 


\section{Disclosure}

The authors report no conflicts of interest in this work.

\section{References}

1. Stern EP, Denton CP. The pathogenesis of systemic sclerosis. Rheum Dis Clin North Am. 2015;41(3):367-382.

2. Ennis H, Vail A, Wragg E, et al. A prospective study of systemic sclerosis-related digital ulcers: prevalence, location, and functional impact. Scand J Rheumatol. 2013;42(6):483-486.

3. Hachulla E, Clerson P, Launay D, et al. Natural history of ischemic digital ulcers in systemic sclerosis: single-center retrospective longitudinal study. J Rheumatol. 2007;34(12):2423-2430.

4. Mihai C, Landewé R, van der Heijde D, et al; EUSTAR Co-Authors. Digital ulcers predict a worse disease course in patients with systemic sclerosis. Ann Rheum Dis. Epub 2015 Feb 16.

5. Mouthon L, Carpentier PH, Lok C, et al; ECLIPSE Study Investigators. Ischemic digital ulcers affect hand disability and pain in systemic sclerosis. J Rheumatol. 2014;41(7):1317-1323.

6. Brand M, Hollaender R, Rosenberg D, et al; EUSTAR Co-Investigators. An observational cohort study of patients with newly diagnosed digital ulcer disease secondary to systemic sclerosis registered in the EUSTAR database. Clin Exp Rheumatol. 2015;33(4 suppl 91):S47-S54.

7. Mouthon L, Mestre-Stanislas C, Bérezné A, et al. Impact of digital ulcers on disability and health-related quality of life in systemic sclerosis. Ann Rheum Dis. 2010;69(1):214-217.

8. Bérezné A, Seror R, Morell-Dubois S, et al. Impact of systemic sclerosis on occupational and professional activity with attention to patients with digital ulcers. Arthritis Care Res (Hoboken). 2011;63(2):277-285.

9. Merkel PA, Herlyn K, Martin RW, et al; Scleroderma Clinical Trials Consortium. Measuring disease activity and functional status in patients with scleroderma and Raynaud's phenomenon. Arthritis Rheum. 2002;46:2410-2420.

10. Gualtierotti R, Adorni G, Lubatti C, Zeni S, Meroni PL, Ingegnoli F. Digital ulcer management in patients with systemic sclerosis. $O A$ Arthritis. 2014;18(1):2.

11. Di Franco M, Bazzichi L, Casale R, Sarzi-Puttini P, Atzeni F. Pain in systemic connective tissue diseases. Best Pract Res Clin Rheumatol. 2015;29(1):53-62.
12. Giuggioli D, Manfredi A, Colaci M, Ferri C. Oxycodone in the longterm treatment of chronic pain related to scleroderma skin ulcers. Pain Med. 2010;11(10):1500-1503.

13. Bonezzi C, Nava A, Barbieri M, et al. Validazione della versione italiana del Brief Pain Inventory nei pazienti con dolore cronico [Validation of the Italian version of Brief Pain Inventory in patients with chronic pain]. Minerva Anestesiol. 2002;68(7-8):607-611.

14. Gualtierotti R, Ingegnoli F, Two R, et al; VERITAS Study Group. Reliability and validity of the Italian version of the UCLA Scleroderma Clinical Trial Consortium Gastrointestinal Tract Instrument in patients with systemic sclerosis. Clin Exp Rheumatol. 2015;33(4 suppl 91): S55-S60.

15. Khanna D, Hays RD, Maranian P, et al. Reliability and validity of the University of California, Los Angeles Scleroderma Clinical Trial Consortium Gastrointestinal Tract Instrument. Arthritis Rheum. 2009;61(9): 1257-1263.

16. Chou R, Fanciullo GJ, Fine PG, et al; American Pain Society-American Academy of Pain Medicine Opioids Guidelines Panel. Clinical guidelines for the use of chronic opioid therapy in chronic noncancer pain. J Pain. 2009;10(2):113-130.

17. Guerriero F, Maurizi N, Francis M, et al. Is oxycodone/naloxone effective and safe in managing chronic pain of a fragile elderly patient with multiple skin ulcers of the lower limbs? A case report. Clin Interv Aging. 2015;10:1283-1287.

18. Guseva NG, Alekperov RT, Anikina NV. Tramadol in the vascular pains of patients with systemic scleroderma and other rheumatic diseases. Ter Arkh. 1994;66:20-24.

19. Poelaert J, Koopmans-Klein G, Dioh A, et al. Treatment with prolongedrelease oxycodone/naloxone improves pain relief and opioid-induced constipation compared with prolonged-release oxycodone in patients with chronic severe pain and laxative-refractory constipation. Clin Ther. 2015;37(4):784-792.

20. Valenzuela A, Chung L. Calcinosis: pathophysiology and management. Curr Opin Rheumatol. 2015;27(6):542-548.

21. van Ingen ILA, Jansen MM, Barrera P. Topical opioids for painful ulcers in systemic sclerosis. Ann Rheum Dis. 2008;67:427.

22. Giuggioli D, Manfredi A, Vacchi C, Sebastiani M, Spinella A, Ferri C. Procedural pain management in the treatment of scleroderma digital ulcers. Clin Exp Rheumatol. 2015;33(1):5-10.
Clinical Interventions in Aging

\section{Publish your work in this journal}

Clinical Interventions in Aging is an international, peer-reviewed journal focusing on evidence-based reports on the value or lack thereof of treatments intended to prevent or delay the onset of maladaptive correlates of aging in human beings. This journal is indexed on PubMed Central, MedLine,

\section{Dovepress}

CAS, Scopus and the Elsevier Bibliographic databases. The manuscript management system is completely online and includes a very quick and fair peer-review system, which is all easy to use. Visit http://www.dovepress. com/testimonials.php to read real quotes from published authors 